\title{
KLOE measurement of the charged kaon absolute semileptonic BR's
}

\author{
Barbara Sciascia*t \\ INFN Frascati, Italy \\ E-mail: barbara.sciascia@lnf.infn.it
}

This paper is devoted to the measurement of the fully inclusive of photon radiation absolute branching ratios of the semileptonic charged-kaon decays, $K^{ \pm} \rightarrow \pi^{0} e^{ \pm} v(\gamma)$ and $K^{ \pm} \rightarrow$ $\pi^{0} \mu^{ \pm} v(\gamma)$. The measurements have been done using a tag technique, via identification of the twobody decays $K^{ \pm} \rightarrow \mu^{ \pm} v$ and $K^{ \pm} \rightarrow \pi^{ \pm} \pi^{0}$, and using a sample of about $410 \mathrm{pb}^{-1}$ collected during the 2001 and 2002 data taking of the KLOE experiment at DA $\Phi$ NE, the Frascati $\phi$ factory. The results obtained are $B R\left(K_{e 3}\right)=0.04965(38)_{\text {Stat }}(37)_{\text {Syst }}$ and $B R\left(K_{\mu 3}\right)=0.03233(29)_{\text {Stat }}(26)_{\text {Syst }}$.

Kaon International Conference

May 21-25, 2007

Laboratori Nazionali di Frascati dell'INFN

\footnotetext{
* Speaker.

$\dagger$ for the KLOE collaboration: F. Ambrosino, A. Antonelli, M. Antonelli, F. Archilli, C. Bacci, P. Beltrame, G. Bencivenni, S. Bertolucci, C. Bini, C. Bloise, S. Bocchetta, V. Bocci, F. Bossi, P. Branchini, R. Caloi, P. Campana, G. Capon, T. Capussela, F. Ceradini, S. Chi, G. Chiefari, P. Ciambrone, E. De Lucia, A. De Santis, P. De Simone, G. De Zorzi, A. Denig, A. Di Domenico, C. Di Donato, S. Di Falco, B. Di Micco, A. Doria, M. Dreucci, G. Felici, A. Ferrari, M. L. Ferrer, G. Finocchiaro, S. Fiore, C. Forti, P. Franzini, C. Gatti, P. Gauzzi, S. Giovannella, E. Gorini, E. Graziani, M. Incagli, W. Kluge, V. Kulikov, F. Lacava, G. Lanfranchi, J. Lee-Franzini, D. Leone, M. Martini, P. Massarotti, W. Mei, L. Meola, S. Miscetti, M. Moulson, S. Müller, F. Murtas, M. Napolitano, F. Nguyen, M. Palutan, E. Pasqualucci, A. Passeri, V. Patera, F. Perfetto, M. Primavera, P. Santangelo, G. Saracino, B. Sciascia, A. Sciubba, F. Scuri, I. Sfiligoi, A. Sibidanov, T. Spadaro, M. Testa, L. Tortora, P. Valente, B. Valeriani, G. Venanzoni, R. Versaci, G. Xu
} 
The most precise test of unitarity of the CKM matrix comes from its first row: $1-\Delta \simeq\left|V_{u d}\right|^{2}+$ $\left|V_{u s}\right|^{2}$. Using $V_{\mathrm{ud}}$ as extracted from nuclear beta decays, and $V_{\mathrm{us}}$ as extracted from semileptonic kaon decay width, a precision test on $\Delta$ with e precision of few parts per mil can be performed. With the KLOE detector we can measure all experimental inputs to $V_{\mathrm{us}}$ : branching ratios, lifetimes, and form factors. Here we report about the measurement of the absolute branching ratios of the semileptonic charged-kaon decays, $K^{ \pm} \rightarrow \pi^{0} e^{ \pm} v(\gamma)\left(K_{e 3}^{ \pm}\right)$and $K^{ \pm} \rightarrow \pi^{0} \mu^{ \pm} v(\gamma)\left(K_{\mu 3}^{ \pm}\right)$, using a sample of about $410 \mathrm{pb}^{-1}$ collected during the 2001 and 2002 data taking.

The first section (1) brefly describes the accelerator DAФNE and the KLOE detector. The measurements have been done using a tag technique presented in section 2 . The following two sections are dedicated to the selection of the tag samples (3) and the signal sample (4) respectively. MC efficiency has to be corrected using suitable Data and MC control samples, as described in section 5. The results are summarized in section 6, while the last section (7) contains information about the $V_{\mathrm{us}}$ extraction and the lepton flavour violation test, using all the $K_{13} \mathrm{KLOE}$ results.

\section{DA $\Phi N E$ and KLOE}

In the DA $\Phi N E e^{+} e^{-}$collider, beams collide at a center-of-mass energy $W \sim M(\phi)$. Since 2001, KLOE has collected an integrated luminosity of $\sim 2.5 \mathrm{fb}^{-1}$. Results presented below are based on 2001-02 data for $\sim 410 \mathrm{pb}^{-1}$. The KLOE detector consists of a large cylindrical drift chamber surrounded by a lead/scintillating-fiber electromagnetic calorimeter. A superconducting coil around the calorimeter provides a $0.52 \mathrm{~T}$ field. The drift chamber [1] is $4 \mathrm{~m}$ in diameter and $3.3 \mathrm{~m}$ long. The momentum resolution is $\sigma\left(p_{T}\right) / p_{T} \sim 0.4 \%$. Two track vertices are reconstructed with a spatial resolution of $\sim 3 \mathrm{~mm}$. The calorimeter [2] composed of a barrel and two endcaps, covers $98 \%$ of the solid angle. Energy and time resolution are $\sigma(E) / E=5.7 \% / \sqrt{E(\mathrm{GeV})}$ and $\sigma(t)=57 \mathrm{ps} / \sqrt{E(\mathrm{GeV})} \oplus 100 \mathrm{ps}$. The KLOE trigger [3] uses calorimeter and drift chamber information. For the present analysis only the calorimeter signals are used. Two energy deposits above threshold, $E>50 \mathrm{MeV}$ for the barrel and $E>150 \mathrm{MeV}$ for the endcaps, are required.

\section{Method of measurement}

The $\phi$ meson decays mainly into kaons: $49 \%$ to $K^{+} K^{-}$and $34 \%$ to $K_{L}-K_{S}$ pairs. The identification of the $K^{+}$automatically tags the presence of a $K^{-}$; the same holds with reversed charges. In KLOE $K^{ \pm}$decays are tagged efficiently by the identification of a two body decay of one of the kaons. Both $K^{ \pm} \rightarrow \mu^{ \pm} v\left(K_{\mu 2}^{ \pm}\right)$and $K^{ \pm} \rightarrow \pi^{ \pm} \pi^{0}\left(K_{\pi 2}^{ \pm}\right)$decays have been used for the measurements presented in this paper.

The use of a tagging technique gives the possibility to perform absolute branching ratio measurements. The branching ratio of the signal decay can be extracted from:

$$
B R(\operatorname{Sig})=\frac{N_{S i g}}{N_{T a g} \varepsilon_{F V} \varepsilon_{S i g}} \frac{\sum_{i} B R(i) \varepsilon_{T a g}(i)}{\varepsilon_{T a g}(S i g)},
$$

where $\varepsilon_{F V}$ is the geometrical acceptance of the fiducial volume, and $\varepsilon_{S i g}$ is the signal reconstruction and selection efficiency. $\varepsilon_{S i g}$ is measured directly using the $\mathrm{MC}$, and corrected for the different 
behaviors for Data and MC of quantities involved in the measurement like the tracking efficiency or the single photon efficiency.

In principle, the capability of selecting a tag kaon does not depend on the decay mode of the other kaon. In fact the geometrical superimposition of the "tag" and "signal" part of the $K^{+} K^{-}$ event, and the fact that the trigger and the background rejection and tracking procedures look at the event globally, make the separation in two distinct hemispheres arbitrary. In short, the $K^{ \pm}$tagging efficiency is not independent of the $K^{\mp}$ decay mode, and the tag bias has to be therefore carefully studied. The factor

$$
\frac{\sum_{i} B R(i) \varepsilon_{T a g}(i)}{\varepsilon_{T a g}(S i g)}
$$

should be equal to 1 , if for each decay mode, $i$, the efficiency $\varepsilon(i)$ is the same.The above quantity is referred to as the tag bias and can be measured only using MC. The correction due to the different behaviors for Data and MC of specific processes or sub-detectors has been measured in suitable dowscaled event samples.

\section{Tag selection}

The measurement of the branching ratios for the $K^{ \pm}$semileptonic decays is performed using four data samples defined by different decay modes for the tagging kaon: $K_{\mu 2}^{+}, K_{\pi 2}^{+}, K_{\mu 2}^{-}$, and $K_{\pi 2}^{-}$. This redundancy allows the systematic effects due to the tag selection to be kept under control.

Tagging kaons are identified as tracks with momentum $70<p_{K}<130 \mathrm{MeV}$, originating from the $e^{+} e^{-}$collision point (I.P.). The kaon decay vertex must be within a fiducial volume (FV) defined as a cylinder of radius $40<r<150 \mathrm{~cm}$, and height $|\mathrm{z}|<130 \mathrm{~cm}$, centered at the I.P., coaxial with the beams. The decay track, extrapolated to the calorimeter, must point to an appropriate energy deposit. $K_{\mu 2}^{ \pm}\left(K_{\pi 2}^{ \pm}\right)$decays are selected by applying a $3 \sigma$ cuts around the muon (pion) momentum calculated in the kaon rest frame, according to the proper mass hypothesis. For the $K_{\pi 2}^{ \pm}$ tag, identification of the $\pi^{0}$ from the vertex is also required. Finally, to reduce the dependency of the tag selection efficiency on the decay mode of the signal kaon, the energy deposits associated to the tagging decay, are required to satisfy the calorimeter trigger. In the analyzed data set about 60 million tag decays were identified and divided into the four tag samples.

The value of the tag bias, which accounts for the capability to separate each event into a "tag" and a "signal" part, is shown in table 1 separately per tag and per signal modes. This correction ranges from about $-3 \%$ to $+4 \%$ following the tag sample used.

\begin{tabular}{c|c|c|c|c} 
& $K_{\mu 2}^{+}$ & $K_{\pi 2}^{+}$ & $K_{\mu 2}^{-}$ & $K_{\pi 2}^{-}$ \\
\hline$K_{e 3}$ & $0.9694(11)(49)$ & $1.0137(34)(52)$ & $0.9884(10)(47)$ & $1.0328(23)(32)$ \\
$K_{\mu 3}$ & $0.9756(13)(50)$ & $1.0210(36)(52)$ & $0.9963(10)(48)$ & $1.0371(25)(32)$
\end{tabular}

Table 1: Tag bias per tag and per signal type, measured in MC and corrected for different Data-MC behavior Statistical and systematic errors are shown. 


\section{Selection of semileptonic $K^{ \pm}$decays}

The reconstruction of a one-prong kaon decay vertex (performed with the same requests used in the identification of the tag) is the first step of the selection followed by the identification of a $\pi^{0}$ associated to the decay vertex using a time of flight technique. In doing this, for each cluster not associated to a track, the kaon decay time, $t_{i}$, is calculated using the cluster time, $t_{c l u}^{i}$, and the distance, $L_{i}$, between the vertex and the cluster position: $t_{i}=t_{c l u}^{i}-L_{i} / c$. This time should be the same for two photons coming from a $\pi^{0}$ decay, and at least a pair of clusters has to satisfy the condition $t_{1}-t_{2}<3 \sigma_{t}\left(\sigma_{t}=\sigma_{t, 1} \oplus \sigma_{t, 2}\right)$. Using the energy and the position of the clusters of the selected pair, the $\gamma \gamma$ invariant mass is calculated and a $3 \sigma$ cut $(\sigma \sim 18 \mathrm{MeV})$ is applied around the nominal $\pi^{0}$ mass. This cut is intended to remove events with accidental coincidence of clusters due to machine background clusters entering the calorimeter.

To isolate the $K_{e 3}$ and $K_{\mu 3}$ decays, the lepton is identified by a time of flight technique. This requires that the lepton track is extrapolated to the calorimeter and geometrically associated to a calorimeter cluster. Specifically, the kaon decay time estimated from the $\pi^{0}$ photons $\left(t_{\pi^{0}}^{\text {decay }}\right)$ should be equal to the one estimated from the charged track $\left(t_{\text {lept }}^{\text {decay }}\right)$, if the correct mass has been assigned to it. The kaon decay time, estimated from $\pi^{0}$ photons, is: $t_{\pi^{0}}^{\text {decay }}=t_{i}-L_{i} / c$. For the lepton the proper track length and velocity are used: $t_{\text {lept }}^{\text {decay }}=t_{\text {lept }}-\frac{L_{\text {lept }}}{p_{\text {lept }} c} \sqrt{p_{\text {lept }}^{2}+m_{\text {lept }}^{2}}$, where $t_{\text {lept }}$ is the time of the cluster associated to the secondary track, and $p_{\text {lept }}$ and $L_{\text {lept }}$ are the momentum, measured in the laboratory rest frame, and the length of this track respectively. The lepton mass is then obtained, imposing $t_{\pi^{0}}^{\text {decay }}=t_{\text {lept }}^{\text {decay }}$ :

$$
m_{\text {lept }}^{2}=p_{\text {lept }}^{2} \cdot\left[\frac{c^{2}}{L_{\text {lept }}^{2}}\left(t_{\text {lept }}-t_{\pi^{0}}^{\text {decay }}\right)^{2}-1\right] .
$$

To fight against the more abundant two body decays, the momentum of the secondary track is computed in the kaon rest frame, using the pion mass hypothesis. All events with $p_{\pi}^{*}>195 \mathrm{MeV}$ are rejected. Only poorly reconstructed $K_{\mu 2}$ and $K_{\pi 2}$ kinks, or $K_{\pi 2}$ events with an early $\pi^{ \pm}$decay, survive this cut. These last events are rejected imposing to the lepton momentum $\left(p_{\mu}^{*}\right)$ calculated in the center of mass of the $\pi^{ \pm}$(defined as missing momentum at the decay vertex, $P_{K}-p_{\pi^{0}}$ ), the condition $p_{\mu}^{*}>60 \mathrm{MeV}$. After all selection cuts, the contamination from non- $K_{13}$ events is less than $\sim 1.5 \%$ in each tag sample. To obtain the number of signal events, a constrained likelihood fit is applied to the $m_{\text {lept }}^{2}$ data spectrum using a linear combination of $K_{e 3}$ and $K_{\mu 3}$ shapes, and of the background contribution. $\mathrm{MC}$ shapes are corrected for Data/MC differences on the calorimeter timing. About $300000 K_{e 3}$ and $160000 K_{\mu 3}$ have been found in the 2001-2002 data set.

In figure 1, the fit results of $m_{\text {lept }}^{2}$ distribution for the $K_{\pi 2}^{+}$tag sample is shown. The $K_{e 3}$ signal component is evident as a narrow peak around zero, well separated from the background. The $K_{\mu 3}$ signal is the peak around the $m_{\mu}^{2}$ value. Other tag samples show the same behavior.

\section{Efficiency evaluation}

The selection efficiency is measured on MC and corrected for relevant Data/MC differences, in particular the tracking efficiency and the calorimeter clustering efficiency, for both photons and 


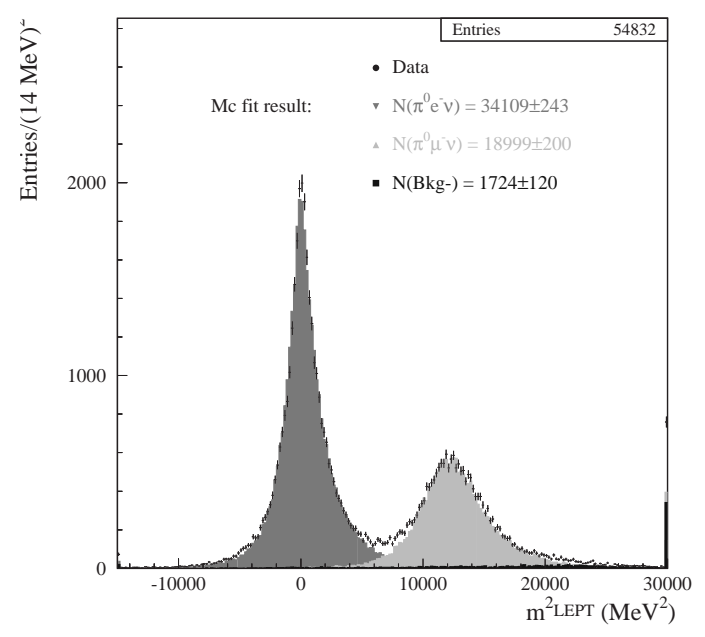

Figure 1: Fit results of $m_{\text {lept }}^{2}$ distribution for the $K_{\pi 2}^{+}$tag sample.

leptons. For each correction, a control sample has been selected in such a way that the efficiency can be measured as function of a suitable set of variables.

For the tracking, a kinematic fit applied to a $K_{13}$ sample -statistically independent from the one used for the measurement- has been used to extract the tracking efficiency as a function of the kaon polar angle, the decay vertex position, and the lepton momentum. The photon cluster efficiency has been measured as a function of the photon energy using a sample of $K_{\pi 2}^{ \pm}$decays. A $K_{L e 3}$ event sample has been used to obtain the electron cluster efficiency as a function of the lepton momentum and the incidence angle on the EMC. Finally the muon cluster efficiency has been extracted, as a function of the same variables of the electron, using a combined sample of $K_{L \mu 3}, K_{\pi 2}^{ \pm}$(with a well reconstructed $\pi \rightarrow \mu$ kink), and $K_{\mu 2}^{ \pm}$events.

All the data/MC corrections are stable with respect to the variation of the cuts applied in selecting the control samples.

\section{Results}

For all the relevant analysis aspects, the systematic errors have been carefully evaluated for each tag sample and for each decay, taking correlations into account. All contributions to the final error, both statistical and systematic, are summarized in table 2 for both $K_{e 3}$ and $K_{\mu 3}$ measurements. The final errors are dominated by the statistical error of the efficiency corrections: the tracking is the dominant contribution for the $K_{e 3}$ measurement, while for $K_{\mu 3}$, tracking and muon cluster corrections are at the same level. Final fractional accuracy ranges, depending on the tag sample, from $1.5 \%$ to $2.1 \%$ for the $K_{e 3}$, and from $1.5 \%$ to $2.7 \%$ for the $K_{\mu 3}$ measurements.

The $\chi^{2}$ for the 4 independent-tag measurements is $1.62 / 3$, with a probability of about $65 \%$ for the $K_{e 3}$, and $1.07 / 3$, with a probability of about $78 \%$ for the $K_{\mu 3}$ decays. The averages of the four 


\begin{tabular}{|c|c|c|c|c|c|c|c|c|}
\hline & \multicolumn{4}{|c|}{$K_{e 3}$} & \multicolumn{4}{|c|}{$K_{\mu 3}$} \\
\hline \multirow[t]{2}{*}{ Source } & $K_{\mu 2}^{+}$ & $K_{\pi 2}^{+}$ & $K_{\mu 2}^{-}$ & $K_{\pi 2}^{-}$ & $K_{\mu 2}^{+}$ & $K_{\pi 2}^{+}$ & $K_{\mu 2}^{-}$ & $K_{\pi 2}^{-}$ \\
\hline & \multicolumn{8}{|c|}{ Statistical (\%) } \\
\hline Tag bias (TB) & 0.07 & 0.14 & 0.08 & 0.14 & 0.09 & 0.18 & 0.09 & 0.17 \\
\hline Cosmic veto corr. to $\mathrm{TB}$ & 0.00 & 0.01 & 0.01 & 0.01 & 0.01 & 0.01 & 0.01 & 0.01 \\
\hline Machine Bkg corr. to TB & 0.09 & 0.31 & 0.05 & 0.17 & 0.09 & 0.31 & 0.05 & 0.17 \\
\hline Nuclear int. corr. & 0.32 & 0.57 & - & - & 0.32 & 0.57 & - & - \\
\hline Fit counting & 0.40 & 0.71 & 0.40 & 0.72 & 0.61 & 1.05 & 0.60 & 1.08 \\
\hline \multirow[t]{2}{*}{$\varepsilon$ correction } & 1.17 & 1.67 & 1.24 & 1.78 & 1.61 & 2.25 & 1.12 & 2.27 \\
\hline & \multicolumn{8}{|c|}{ Systematics: signal (\%) } \\
\hline$\varepsilon$ corr. (TRK) & 0.54 & 0.54 & 0.53 & 0.53 & 0.44 & 0.43 & 0.43 & 0.43 \\
\hline$\varepsilon$ corr. (lepton clus.) & 0.00 & 0.00 & 0.00 & 0.00 & 0.14 & 0.14 & 0.14 & 0.14 \\
\hline$\varepsilon$ corr. $\left(\pi^{0}\right)$ & 0.24 & 0.25 & 0.24 & 0.24 & 0.21 & 0.21 & 0.21 & 0.21 \\
\hline Fit & 0.13 & 0.19 & 0.35 & 0.15 & 0.03 & 0.16 & 0.19 & 0.06 \\
\hline \multirow[t]{2}{*}{ Selection cuts } & 0.17 & 0.17 & 0.17 & 0.16 & 0.49 & 0.49 & 0.49 & 0.48 \\
\hline & \multicolumn{8}{|c|}{ Systematics: acceptance (\%) } \\
\hline Nuclear int. corr. & 0.18 & 0.39 & - & - & 0.18 & 0.39 & - & - \\
\hline \multirow[t]{2}{*}{$\tau_{ \pm}$} & 0.09 & 0.09 & 0.09 & 0.09 & 0.09 & 0.09 & 0.09 & 0.09 \\
\hline & \multicolumn{8}{|c|}{ Systematics: tag bias corrections (\%) } \\
\hline Machine Bkg & 0.36 & 0.06 & 0.37 & 0.05 & 0.36 & 0.06 & 0.37 & 0.05 \\
\hline Cosmic veto & 0.04 & 0.02 & 0.03 & 0.04 & 0.04 & 0.02 & 0.03 & 0.04 \\
\hline \multirow[t]{3}{*}{ Nuclear int. } & 0.09 & 0.13 & - & - & 0.09 & 0.13 & - & - \\
\hline & \multicolumn{8}{|c|}{ Total $(\%)$} \\
\hline & 1.49 & 2.08 & 1.54 & 2.03 & 1.95 & 2.71 & 1.52 & 2.63 \\
\hline
\end{tabular}

Table 2: Summary of all fractional contributions to the error on $K_{e 3}$ and $K_{\mu 3}$ branching ratio measurements.

results are:

$$
\begin{aligned}
\mathrm{BR}\left(\mathrm{K}^{-} \rightarrow \pi^{0} \mathrm{e}^{-} v\right) & =\left(4.946 \pm 0.053_{\text {Stat }} \pm 0.038_{\text {Syst }}\right) \times 10^{-2} \\
\mathrm{BR}\left(\mathrm{K}^{+} \rightarrow \pi^{0} \mathrm{e}^{+} v\right) & =\left(4.985 \pm 0.054_{\text {Stat }} \pm 0.037_{\text {Syst }}\right) \times 10^{-2} \\
\mathrm{BR}\left(\mathrm{K} \rightarrow \pi^{0} \mathrm{e} v\right) & =\left(4.965 \pm 0.038_{\text {Stat }} \pm 0.037_{\text {Syst }}\right) \times 10^{-2},
\end{aligned}
$$

for the $K_{e 3}$, and

$$
\begin{aligned}
\mathrm{BR}\left(\mathrm{K}^{-} \rightarrow \pi^{0} \mu^{-} v\right) & =\left(3.219 \pm 0.047_{\text {Stat }} \pm 0.027_{\text {Syst }}\right) \times 10^{-2} \\
\mathrm{BR}\left(\mathrm{K}^{+} \rightarrow \pi^{0} \mu^{+} v\right) & =\left(3.241 \pm 0.037_{\text {Stat }} \pm 0.026_{\text {Syst }}\right) \times 10^{-2} \\
\mathrm{BR}\left(\mathrm{K} \rightarrow \pi^{0} \mu v\right) & =\left(3.233 \pm 0.029_{\text {Stat }} \pm 0.026_{\text {Syst }}\right) \times 10^{-2}
\end{aligned}
$$

for the $K_{\mu 3}$. The $\chi^{2}$ between different charge measurements is $0.17 / 1$, with a probability of about $68 \%$ for the $K_{e 3}$, and $0.12 / 1$, with a probability of about $73 \%$ for the $K_{\mu 3}$ decays. Final BR results have a fractional accuracy of $1.1 \%$ for the $K_{e 3}$ and of $1.2 \%$ for the $K_{\mu 3}$ decays. 
The $\tau_{K}$ value affects the $\mathrm{BR}$ values via the geometrical acceptance evaluation. From suitable MC sample the BR dependency on the $\tau_{K}$ has been estimated: $\operatorname{BR}\left(K_{13}, \tau\right)=\operatorname{BR}\left(K_{13}, \tau^{0}\right) \cdot\left(1-0.45\left(\tau_{K^{-}}\right.\right.$ $\left.\left.\tau^{0}\right) / \tau^{0}\right)$, with $\tau^{0}=12.36 \mathrm{~ns}, \operatorname{BR}\left(K_{e 3}, \tau^{0}\right)=4.968 \%$, and $\operatorname{BR}\left(K_{\mu 3}, \tau^{0}\right)=3.234 \%$. The final results are evaluated using the PDG06 [5] fit value $\tau=12.384(24)$ ns.

While the correlation between the $K_{e 3}$ and $K_{\mu 3}$ signals induced by the fit procedure is low (about $1 \%$ ), a correlation is caused by the corrections to the tag bias, which are equal for the two signals, by the same Data/MC corrections for the tracking and the clustering, and finally by the selection cuts. Excluding the error coming from $\tau_{K}$ value, the complete information is contained in the error matrix:

$$
\left(\begin{array}{ll}
0.2780 & 0.1268 \\
0.1268 & 0.1510
\end{array}\right) \times 10^{-6},
$$

from which a $62.74 \%$ of correlation between $K_{e 3}$ and $K_{\mu 3}$ can be extracted.

For $K_{\mu 3}$ only also the dependency on $\lambda_{0}$ has to be considered. The final $B R\left(K_{\mu 3}\right)$ has been evaluated using $\lambda_{0} \sim 0.015$ and the relation $B R\left(K_{\mu 3}\right)=0.0327-0.0230 \lambda_{0}$, obtained from a dedicated MC study. The limited knowledge of $\lambda_{0}$ value gives a negligible contribution to the systematic error.

The BR are completely inclusive of the photon radiation. The radiated photon acceptance is determined with a generator that uses the soft-photon approximation to sum the amplitudes for real and virtual processes to all order of $\alpha[6]$.

The ratio $R_{\mu e}=\Gamma\left(K_{\mu 3}\right) / \Gamma\left(K_{e 3}\right)$ has been measured in the four tag samples used for the BR measurements. With respect to the $\mathrm{BR}, R_{\mu e}$ benefits of smaller tag bias corrections ranging from $0.4 \%$ to $0.8 \%$ depending on the tag sample. The correlation between $K_{e 3}$ and $K_{\mu 3}$ coming from the fit procedure and from the efficiency corrections has been taken into account in calculating the error on $R_{\mu e}$. As in the BR determination, the errors are dominated by the statistics of the efficiency corrections. The average of the four tag sample measurements is $R_{\mu e}^{K L O E}=0.6511(46)_{\text {Stat }}(73)_{\text {Syst }}$. This measurement has a fractional error of about $1.3 \%$ and is in agreement within the errors with the theoretical prediction: $R_{\mu e}^{S M}=\left(1-\delta_{K}^{\mu}\right) /\left(1-\delta_{K}^{e}\right) \cdot\left(I_{K}^{\mu}\right) /\left(I_{K}^{e}\right)=0.6646(61)$, where the integrals $\left(I_{K}^{e}\right.$ and $\left.I_{K}^{\mu}\right)$ and $\delta_{S U(2)}, \delta_{e m}$ corrections are from [9].

\section{7. $V_{\mathrm{us}}$ determination from KLOE results}

The BR's of the semileptonic $K^{ \pm}$decays, together with the already published results for the semileptonic decays of the $K_{L}[7]$ and $K_{S}$ [8], allow five independent determinations of the observable $\left|V_{\mathrm{us}} f_{+}(0)\right|$, as shown in Fig. 2. All inputs, but $K_{13}$ branching ratios, needed to extract $\left|V_{\text {us }} f_{+}(0)\right|$ are from [9]. The average of the five $\left|V_{\text {us }} f_{+}(0)\right|$ values, taking correlations into account, gives $\left|V_{\mathrm{us}} f_{+}(0)\right|=0.2154(5)$, with $\chi^{2}=4.37 / 4$, and has a probability of $\sim 36 \%$. Using $f_{+}(0)=$ $0.961(8)$ from [10], $V_{\mathrm{us}}$ is $0.2241(19)$. This is compatibile with the Unitarity at $1.5 \sigma$, in fact using $V_{\mathrm{ud}}=0.97377(27)$ [11], $V_{\mathrm{ud}}{ }^{2}+V_{\mathrm{us}}{ }^{2}-1$ is equal to $-0.0015(10)$. $\left|V_{\mathrm{us}} f_{+}(0)\right|$ can be evaluated also by charge state. Using $K_{S, L}$ values we obtain $0.2155(6)$, while using $K^{ \pm}$we obtain $0.2146(12)$. These two determination have a $\chi^{2}$ of $0.48 / 1$ with a probability of $49 \%$. To compare $\left|V_{\text {us }} f_{+}(0)\right|$ values evaluated by charge state, $f_{+}(0)$ has to be corrected for the strong $\mathrm{SU}(2)$-breaking. This correction enhances the $K^{ \pm}$decay widths as parameterized by the form-factor normalization correction $\Delta \mathrm{SU}(2)$. The standard value predicted by the $\chi_{P T}$ is $2.31(22) \%$ [12]. The comparison of $\left|V_{\mathrm{us}} f_{+}(0)\right|$ 


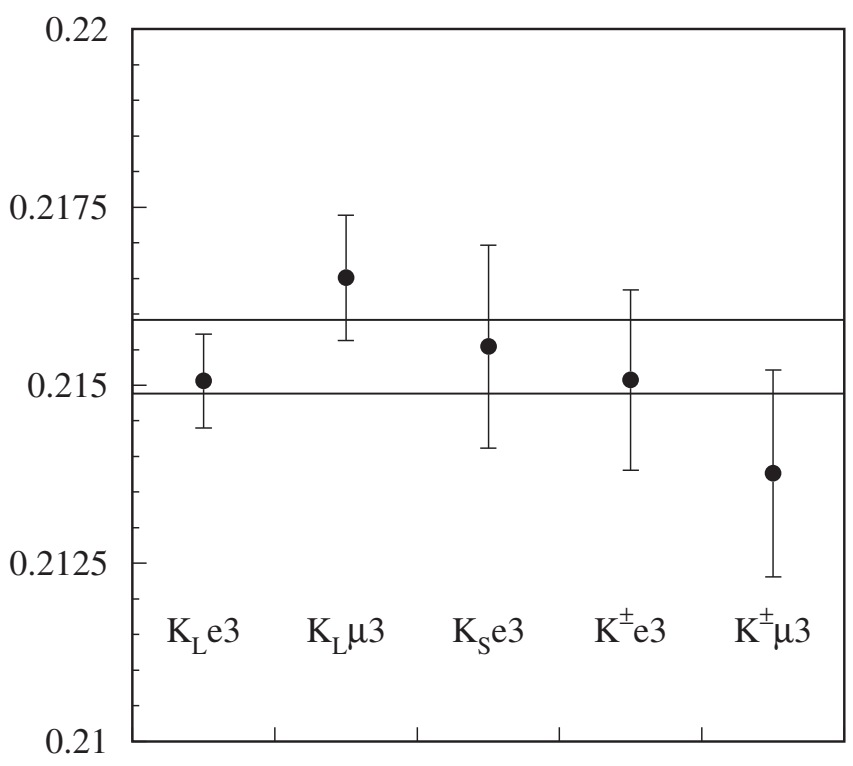

Figure 2: $\left|V_{\mathrm{us}} f_{+}(0)\right|$ measurements.

results by charge state without the application of the $\Delta \mathrm{SU}(2)$ factor, allows an empirical evaluation of the strong SU(2)-breaking, which results to be $1.88(58) \%$, in agreement within the errors with the $\chi_{P T}$ prediction.

Lepton universality can be tested comparing the $R_{\mu e}$ value from the $K_{13}$ measurements, $R_{\mu e}^{O b s}$, and the one from the SM expectations, $R_{\mu e}^{S M}$, defined in the previuos section. Defining $r_{\mu e}=$ $R_{\mu e}^{O b s} / R_{\mu e}^{S M}$, and using the BR KLOE results we obtain for neutral kaon $r_{\mu e}\left(K_{L, S}\right)=1.013(9)$, and for charged kaon $r_{\mu e}\left(K^{ \pm}\right)=0.988(11)$. Both results are compatibile with 1 within the errors, and average to $r_{\mu e}=1.003(7)$, with a $\chi^{2}$ of $3.60 / 1$ and a probability of $5.8 \%$.

\section{References}

[1] M. Adinolfi et al., [KLOE Collaboration], The tracking detector of the KLOE experiment, Nucl. Instrum. Meth A 488200251

[2] M. Adinolfi et al., [KLOE Collaboration], The KLOE electomagnetic calorimeter, Nucl. Instrum. Meth A 4822002364

[3] M. Adinolfi et al., [KLOE Collaboration], The trigger system of the KLOE experiment, Nucl. Instrum. Meth A 4922002134

[4] B. Sciascia, for the KLOE Collab., in Proc. EPS Int.Europhysics Conf. on High-Energy Physics (HEP-EPS '05) (Lisbon 2005), hep-ex 0510028

[5] W.-M. Yao et al., Journal of Physics, G33, 1 (2006)

[6] C. Gatti, Eur.Phys.J.C, 45417,2006

[7] KLOE Collab., F. Ambrosino et al., Phys. Lett. B 632200643 
[8] KLOE Collab., F. Ambrosino et al., Phys. Lett. B 6362006173

[9] M. Moulson for the FlaviaNet Working Group on Kaon Decays, contributed to 4th International Workshop on the CKM Unitarity Triangle, Nagoya, Japan 12-16 Dec 2006, hep-ex/0703013

[10] H. Leutwyler and M. Roos, Z. Phys. C25, 91 (1984)

[11] W. J. Marciano and A. Sirlin, Phys. Rev. Lett., 96: 032002, 2006

[12] V. Cirigliano et al., Eur. Phys. J., C23: 121, 2002 